\title{
The Subjective Size of Melodic Intervals Over a Two-Octave Range
}

Frank A. Russo

University of Toronto

William F. Thompson

University of Toronto

digital.library.ryerson.ca/object/157

Please Cite:

Russo, F. A., \& Thompson, W. F. (2005). The subjective size of melodic intervals over a two-octave range. Psychonomic Bulletin \& Review 12(6), 1068-1075. doi:10.3758/BF03206445

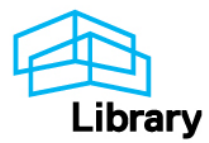




\title{
The subjective size of melodic intervals over a two-octave range
}

\author{
FRANK A. RUSSO and WILLIAM FORDE THOMPSON \\ University of Toronto, Mississauga, Ontario, Canada
}

\begin{abstract}
Musically trained and untrained participants provided magnitude estimates of the size of melodic intervals. Each interval was formed by a sequence of two pitches that differed by between 50 cents (one half of a semitone) and 2,400 cents (two octaves) and was presented in a high or a low pitch register and in an ascending or a descending direction. Estimates were larger for intervals in the high pitch register than for those in the low pitch register and for descending intervals than for ascending intervals. Ascending intervals were perceived as larger than descending intervals when presented in a high pitch register, but descending intervals were perceived as larger than ascending intervals when presented in a low pitch register. For intervals up to an octave in size, differentiation of intervals was greater for trained listeners than for untrained listeners. We discuss the implications for psychophysical pitch scales and models of music perception.
\end{abstract}

Sensitivity to relations between pitches, called relative pitch, is fundamental to music experience and is the basis of the concept of a musical interval. A musical interval is created when two tones are sounded simultaneously or sequentially. They are experienced as large or small and as consonant or dissonant, and for musically trained listeners, they are associated with category labels, such as the perfect fourth, the minor sixth, and the octave. Sequential intervals form the basis of melody and may occur in ascending or descending pitch directions.

Relative pitch allows us to perceive, appreciate, and remember melodies. Large melodic intervals, or leaps, form the basis for gap-fill melodies (Meyer, 1973) and are experienced as a point of accent (Boltz \& Jones, 1986; Drake, Dowling, \& Palmer, 1991; Jones, 1987). Conversely, melodies sound more coherent or cohesive when they consist of a sequence of small intervals (Huron, 2001; Russo, 2002). Interval size may also influence melodic expectancy (Larson \& McAdams, 2004; Margulis, 2005; Narmour, 1990) and grouping (Deliège, 1987; Lerdahl, 1989; Lerdahl \& Jackendoff, 1983). In short, there is a need for research whose aim is to identify factors that influence the experience of interval size. The goal of this study was to investigate relative pitch by obtaining magnitude estimates of interval size and assessing the influence of three factors: musical training, pitch register, and pitch direction (ascending or descending).

Psychophysical models of pitch can be used to make predictions about judgments of interval size. A logarithmic

This research was supported by a discovery grant awarded to the second author from the Natural Sciences and Engineering Research Council of Canada. We thank Bruce Schneider for helpful comments and Jane Campbell for research assistance. Correspondence should be sent to F. A. Russo or W. F. Thompson, Department of Psychology, University of Toronto, Mississauga, ON, L5L 1C6 Canada (e-mail: frusso@utm .utoronto.ca or b.thompson@utoronto.ca). scale describes the most basic model of pitch. Category labels associated with intervals assume a logarithmic relation between pitch height and frequency; that is, intervals spanning the same log frequency are associated with equivalent category labels, regardless of transposition. Listeners with musical training can explicitly label intervals (Killam, Lorton, \& Schubert, 1975; Plomp, Wagenaar, \& Mimpen, 1973). More important, judgments of interval size by musicians are resistant to the influence of context (J. A. Siegel \& W. Siegel, 1977a) and exhibit steplike functions characteristic of categorical perception (J. A. Siegel \& W. Siegel, 1977b; see also Burns \& Campbell, 1994; Burns \& Ward, 1978). These findings imply that estimates of interval size may depend on musical training.

Drawing from early studies of tone perception by Titchener (1905, pp. 232-248), Stevens, Volkmann, and Newman (1937) questioned whether the psychophysical relation between pitch and frequency was logarithmic and used scaling techniques to derive a new scale, called the mel scale. A pure tone of $1000 \mathrm{~Hz}$ at $40 \mathrm{~dB}$ above threshold was first defined as 1,000 mels, and the pitch in mels of other frequencies was determined by asking participants to adjust a comparison tone until it was perceived to be one half of the pitch height of a standard tone (method of fractionation). Although the mel scale and the logarithmic scale are roughly equivalent below $500 \mathrm{~Hz}$, above $500 \mathrm{~Hz}$, the mel scale increases at a slower rate. That is, perceptually equivalent pitch interval sizes (in mels) span progressively smaller frequency ratios with higher and higher transpositions (Stevens et al., 1937; see also Beck \& Shaw, 1961). These results suggest that estimates of interval size may depend on pitch register.

Another psychophysical scaling method, called the method of equal sense distances, involved presenting listeners with a melodic interval and asking them to divide it into four equal pitch interval sizes (Greenwood, 1997; Stevens \& Volkmann, 1940). Results based on this method 
were similar to those based on the method of fractionation, but an asymmetry was revealed. When the interval was ascending (i.e., low tone first), participants set dividing frequencies higher than when the interval was descending. This asymmetry suggests that estimates of interval size depend on pitch direction.

Some researchers have questioned the relevance of psychophysical scales such as the mel scale (Burns, 1999; Krumhansl, 1990, 2000; Shepard, 1999). First, the tasks used to derive the mel scale have ambiguous interpretations (e.g., half as high), and responses to the tasks are not always reliable (Rasch \& Plomp, 1999). It has been argued that the mel scale cannot be derived from other measures of pitch, such as difference limens, critical bands, or equal cochlear distances (Greenwood, 1997, p. 200), and different strategies of deriving the mel scale yield somewhat different functions (Lewis, 1942; but see Schneider, Parker, \& Upenieks, 1982). Second, the ease with which listeners recognize and reproduce simple melodies independently of pitch register implies that frequency ratios are experienced as invariant across transposition (Attneave \& Olson, 1971). Third, the mel scale was derived from judgments of isolated pure tones, which may have limited relevance to the way tones are perceived in a musical context.

Nonetheless, it is difficult to dismiss the data that were used to derive the mel scale. Specifically, they imply that there is a dimension of melody perception that is somewhat independent of the explicit labels associated with musical intervals (Makeig, 1982). This implication motivated us to obtain direct estimates of the size of melodic intervals and to identify some influences on such judgments. Trained and untrained listeners estimated the size of 48 different intervals ranging between 50 cents (onehalf semitone) to 2,400 cents (two octaves) presented in a high or a low pitch register and in an ascending or a descending pitch direction.

We considered the possibility that the ability to discriminate intervals up to an octave might be somewhat different from the ability to discriminate intervals larger than an octave. Melodic intervals larger than an octave are extremely rare in music, suggesting that intervals up to an octave have a privileged perceptual status. Deutsch and colleagues made a similar distinction, observing that it takes more time to recognize intervals larger than an octave than it does to recognize intervals smaller than an octave. They proposed that recognizing intervals larger than an octave involves two stages. In the first stage, one of the interval tones is mentally transposed, so that both tones can be compared within the same octave range. In the second stage, interval recognition takes place (Deutsch, 1969). Support for this hypothesis has been found in studies of melodic recognition (Deutsch, 1972), melodic dictation (Deutsch \& Boulanger, 1984), and tonal memory (Deutsch, 1978a).

\section{METHOD}

\section{Participants}

Twenty-eight students, 14 musically trained and 14 untrained, were recruited from the University of Toronto community. Untrained participants had 2 years or less of musical instruction $(M=0.57$ years, $S E=0.23$ ) and no continued musical activity. They included 10 females and 2 males ranging in age from 17 to 20 years, with a mean age of 18.5 years. Trained participants had 7 years or more of musical instruction $(M=11.07$ years, $S E=0.55)$ and continued musical activity. They included 10 females and 2 males ranging in age from 17 to 25 years, with a mean age of 18.9 years. All the participants were given course credit. No participant reported having abnormal hearing.

\section{Stimuli}

Seventy-three tones were constructed by additive synthesis with Praat software (Boersma \& Weenink, 2004). The fundamental frequency of each tone was separated by 50 cents from its nearest neighbor, falling within the frequency range of $87.3 \mathrm{~Hz}\left(F_{2}\right)$ to $698.5 \mathrm{~Hz}\left(F_{5}\right)$. All the tones consisted of 12 equal-intensity components (the fundamental and the first 11 overtones). Tones were used to create 48 melodic intervals (two-tone sequences) that varied in size (log frequency distance between tones) from 50 cents (one-half semitone) to 2,400 cents (two octaves). Each of the 48 intervals was presented in ascending and descending pitch directions and in low and high pitch registers. The three factors of interval, pitch direction, and pitch register were counterbalanced. Intervals in the low pitch register were centered on $F_{3}(174.6 \mathrm{~Hz})$, and intervals in the high pitch register were centered on $F_{4}(349.2 \mathrm{~Hz})$. For instance, the largest ascending intervals $\left(2,400\right.$ cents) spanned $F_{2}$ to $F_{4}$ in the low pitch register and $F_{3}$ to $F_{5}$ in the high pitch register.

\section{Procedure}

Each participant completed two blocks each of ascending and descending trials for a total of 384 trials (48 intervals $\times 2$ pitch registers $\times 2$ directions $\times 2$ blocks). Block presentation adhered to one of two orders: (1) ascending, descending, descending, ascending or (2) descending, ascending, ascending, descending. The two orders were counterbalanced across participants, and the order of presentation of trials within blocks was randomly determined for each participant.

The participants provided magnitude estimates of the size of each interval on a scale from 1 to 100 . To discourage explicit mapping of known interval categories, a time limit of $5 \mathrm{sec}$ (with a 3-sec warning) was imposed on each estimate. Before testing, the participants were familiarized with examples of the smallest interval (50 cents, defined as 1) and the largest interval (2,400 cents, defined as 100).

\section{RESULTS}

Magnitude estimates were subjected to a mixed-design ANOVA with training as the between-subjects measure and interval, pitch register, and pitch direction as the within-subjects measures. Timed-out trials represented $1.3 \%$ and $1.0 \%$ of the trials for trained and untrained listeners, respectively. In the event that a trial was timed out, the median rating for the trial corresponding to the listener's training group was substituted for the missing rating.

The main effect of interval was highly significant $[F(47,1222)=124.38, p<.0001]$, with estimates of interval size increasing with increases in the number of semitones separating the two pitches. Figures $1 \mathrm{~A}$ and $1 \mathrm{~B}$ are scatterplots of mean estimates of interval size by untrained and trained listeners, respectively, as a function of the size of intervals in semitones. For untrained and trained listeners, mean estimates of interval size (collapsed across pitch direction) were highly correlated with $\log$ frequency distance $[r(46)=.96$. and 99 , respectively; $p<.0001]$. Despite these high correlations, an inspection 

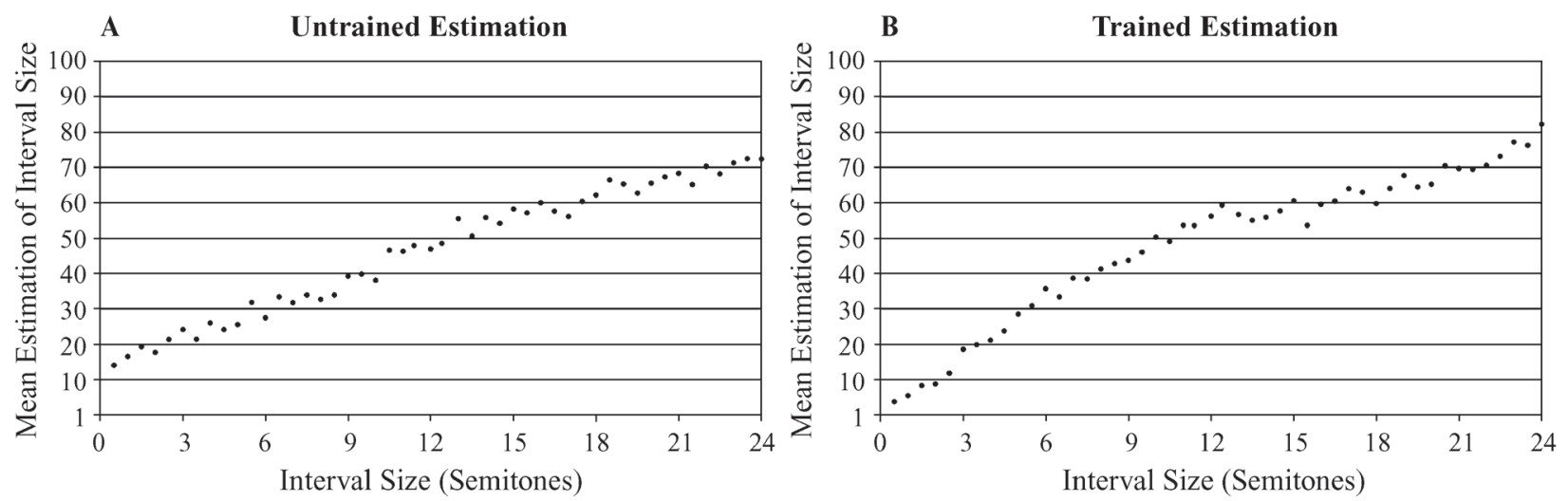

Figure 1. Scatterplots of mean estimates of interval size by (A) untrained listeners and (B) trained listeners as a function of the size of the intervals in semitones.

of the scatterplots suggests that differentiation between intervals up to an octave was greater than differentiation between intervals larger than an octave (particularly for trained listeners). We will explore this effect in greater detail later.

Trained and untrained listeners were dissimilar in the extent to which they experienced intervals as differentiated, leading to a significant two-way interaction between training and interval $[F(47,1222)=2.79, p<.0001]$. Overall, trained listeners experienced greater differentiation, as reflected in a more rapid increase in magnitude estimates with increasing interval size.

Estimates also varied depending on pitch register and pitch direction. Intervals presented in the high pitch register were assigned larger estimates than were intervals presented in the low pitch register $[M=50.88$ and 43.71 , respectively; $F(1,26)=25.55, p<.0001]$. Intervals presented in the descending pitch direction were assigned larger estimates than were intervals presented in the as- cending pitch direction $[M=48.78$ and 45.8 , respectively; $F(1,26)=7.58, p<.05]$. Neither pitch register nor pitch direction interacted with training; however, they did interact with each other $[F(1,26)=104.13, p<.0001]$.

Figure 2 displays mean estimates of interval size for ascending and descending intervals presented in high and low pitch registers. In the high pitch register, larger estimates of interval size were assigned to ascending intervals than to descending intervals $(M=55.02$ and 46.74 , respectively). In the low pitch register, larger estimates of interval size were assigned to descending intervals than to ascending intervals ( $M=50.83$ and 36.58 , respectively). There was also a significant three-way interaction between pitch register, pitch direction, and interval $[F(47,1222)=$ $7.55, p<.0001]$. The interactive effects of pitch register and pitch direction, illustrated in Figure 2, were more evident for larger intervals than for smaller intervals.

To explore the effects of interval in greater detail, regression coefficients representing the linear effects of

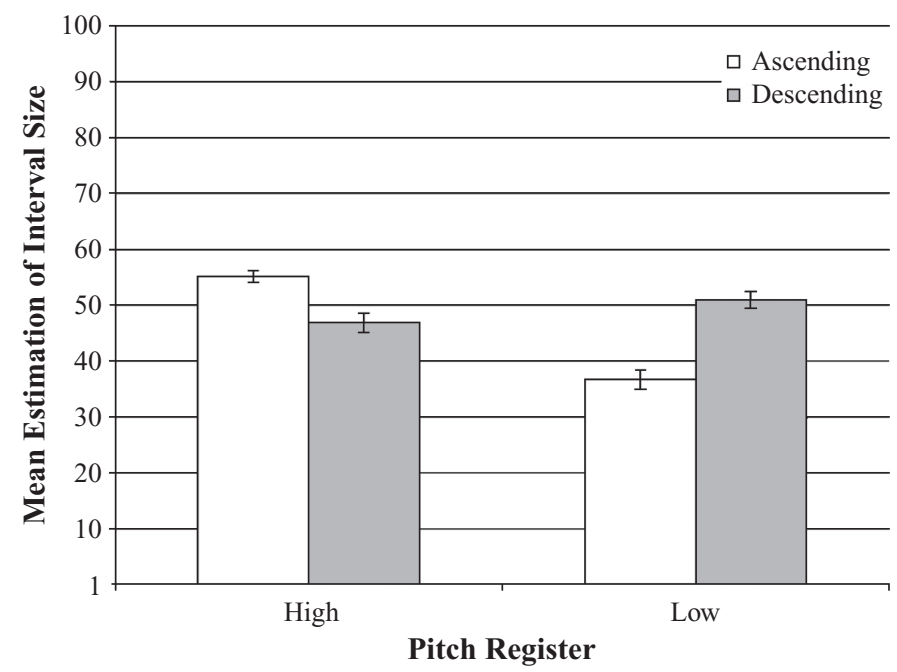

Figure 2. Mean estimates of interval size for ascending and descending intervals presented in high and low pitch registers. 
interval size were computed for intervals up to an octave (small intervals) and intervals larger than an octave (large intervals). Regression coefficients were computed for each participant and each condition and were subjected to a mixed-design ANOVA, with training as the betweensubjects variable and interval set (small or large), pitch register, and pitch direction as the within-subjects variables. ${ }^{1}$

The value of the regression coefficients may be interpreted as a measure of the extent to which listeners differentiated interval sizes. Higher coefficient values (steeper slopes) indicate increased differentiation; lower coefficient values indicate decreased differentiation. Figure 3 plots the mean coefficients for intervals up to an octave (small intervals) and for intervals larger than an octave (large intervals). There was greater differentiation of small intervals than of large intervals $[F(1,26)=191.87$, $p<.0001]$. The difference in slope between small and large intervals was more pronounced for trained listeners than for untrained listeners, leading to an interaction between training and interval set $[F(1,26)=15.61, p<$ $.001]$. Trained listeners showed greater differentiation than did untrained listeners for small intervals $[t(27)=$ $4.635, p<.0001]$, but the two groups showed comparable differentiation for large intervals $[t(27)<1, \mathrm{n} . \mathrm{s}]$.

Regression coefficients also indicate the extent to which listeners mapped equal (logarithmic) changes in fundamental frequency with equal changes in magnitude estimation. Because the smallest and largest intervals were explicitly defined as 1 and 100, respectively, and the largest interval presented was 24 semitones, an optimal mapping of $\log$ frequency onto magnitude estimations should have resulted in a $y$-intercept of 0 and a regression coefficient of $100 \div 24$, or 4.167 . As can be seen in Figure 3, regression coefficients were best predicted by a logarithmic mapping for judgments of small intervals by trained listeners.
The Appendix provides psychologically determined scales of interval size for different levels of pitch register and pitch direction at different levels of musical experience. Each scale was derived from the polynomial function (second order) that best fit mean interval estimates for the 48 intervals tested.

\section{DISCUSSION}

Judgments of interval size increased with increases in the number of semitones between the two tones. However, this association depended on the listener's level of musical training, the pitch register of the interval, the direction of melodic motion, and whether or not the interval was larger than an octave.

For intervals up to an octave, differentiation of intervals was greater for trained than for untrained listeners, as reflected in the slope of the regression lines describing estimates as a function of interval size. Responses by trained listeners were consistent with a logarithmic mapping of fundamental frequency for intervals up to an octave. Presumably, trained listeners are especially sensitive to differences between intervals up to an octave because of their extensive experience with these intervals. However, we cannot rule out the possibility that the observed differences attributed here to training actually reflect underlying differences in predisposition for musical processing (see, e.g., Deutsch, 1978b). Trained listeners may have had an early predisposition for musical processing that led them to pursue formal studies.

For intervals larger than an octave, the slope of the regression lines was similar for trained and untrained listeners. For both groups, regression coefficients suggested less differentiation than was observed for intervals up to an octave. The similarity in results for the two groups suggests that the effects of musical training are not observed

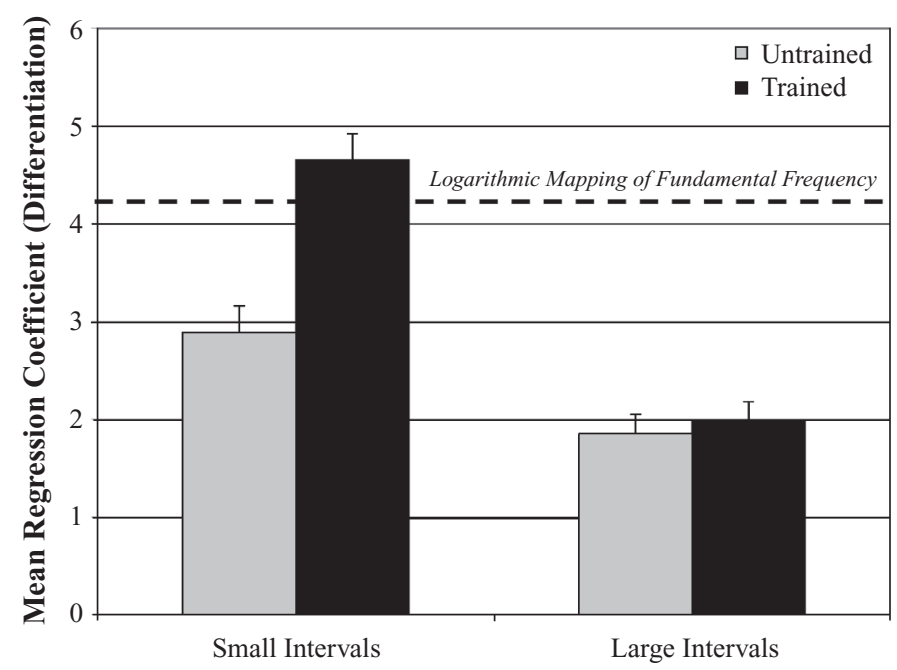

Figure 3. Mean coefficients (interval differentiation) of untrained and trained listeners for small and large intervals. Dotted line represents the logarithmic mapping of frequency onto interval size estimates. 
for intervals larger than an octave, perhaps because such intervals occur infrequently in music. That is, effects of training may be limited to intervals that occur frequently in music and do not generalize to intervals that occur infrequently. A second possibility is that the component tones of intervals larger than an octave were streamed apart at a psychoacoustic level (Bregman, 1990) and that this process occurs independently of musical training. Trained and untrained listeners might differ in their ability to estimate the magnitude of intervals that form a single stream but might not differ in their ability to estimate the magnitude of difference between tones in separate streams.

Intervals presented at a high pitch register were judged to be larger in size than were equivalent intervals presented at a low pitch register. This finding is consistent with predictions based on the mel scale (R. J. Siegel, 1964; Stevens \& Volkmann, 1940; see also Stumpf, 1883). To our knowledge, however, the present study is the first to report an effect of pitch register on direct estimates of interval size for a wide range of intervals that include those found in music. Although our data do not address the question of whether changes in pitch register influence interval recognition or classification, they suggest that identical intervals played at different pitch registers are perceived to be different in size on a phenomenological level.

The mel scale suggests that above $500 \mathrm{~Hz}$, the perceived size of intervals formed by two pure tones should expand with increasing pitch register. The majority of intervals tested in the present study $(76 \%)$ were formed using two complex tones with fundamental frequencies that were below $500 \mathrm{~Hz}$ (i.e., $\mathrm{B}_{4}$ or lower). Indeed, all the intervals up to an octave (small intervals) had component tones below $500 \mathrm{~Hz}$, and in a reanalysis of these intervals, the effect of pitch register persisted $[F(1,26)=10.93, p<$ .01]. Quite possibly, the effect of pitch register was observed because the upper harmonics of all tones tested fell above $500 \mathrm{~Hz}$. This explanation is consistent with research indicating that spectral content influences judgments of interval size (Russo \& Thompson, 2005).

Intervals presented in the descending pitch direction were judged to be larger than equivalent intervals presented in the ascending pitch direction. Statistical analyses of music indicate that descending intervals are smaller, on average, than ascending intervals (Vos \& Troost, 1989; see also Meyer, 1973, p. 145). These statistical regularities may be internalized by listeners and used as standards against which new intervals are interpreted. Thus, a given descending interval may be experienced as larger than an ascending interval of the same size because adaptation levels for interval size predispose listeners to expect descending intervals to be small (Helson, 1964).

There was also a significant interaction between pitch direction and pitch register. In the high pitch register, larger estimates of interval size were assigned to ascending intervals; in the low pitch register, larger estimates of interval size were assigned to descending intervals. Such directional asymmetries have been reported with other methods of psychophysical scaling (Greenwood, 1997). One explanation for this interaction relates to the influ- ence of long-term expectancies. Trained and untrained listeners are sensitive to the pitch range in which pitches and intervals occur (Marley \& Cook, 1984) and may expect melodic intervals to move toward the center of that range. Such expectancies would arise because melodic intervals in music move toward the center of the musical pitch range with greater statistical frequency than they move away from it (von Hippel \& Huron, 2000). ${ }^{2}$ When the initial pitch of a melodic interval is higher than the center of the established pitch range, an ascending interval will bring the second pitch further from the center than will a similarly sized descending interval. Conversely, when the initial pitch of a melodic interval is lower than the center of the musical pitch range, a descending interval will bring the second pitch further from the center than will a similarly sized ascending interval. Movement toward an unexpected event may be perceived as more salient than movement toward an expected event (see also Bartlett, 1993; Bharucha \& Pryor, 1986; Schellenberg, 2001), and these differences in salience may be reflected in estimations of interval size.

A related explanation takes into consideration models of similarity between prototypical and nonprototypical exemplars of categories. Rosch (1975) reported that nonfocal colors are judged to be more similar to focal colors than the other way around. Tversky and Gati (1978) observed comparable asymmetries with prototypic and atypical exemplars of abstract categories. They argued that such asymmetries are due to attentional factors, so that distinctive features of the target are weighted more heavily than distinctive features of the base. For melodic intervals, pitches that fall outside of the expected range of pitch may be perceived as nonprototypical and distinctive, so that movement from a prototypical pitch toward a nonprototypical pitch connotes less similarity between pitches than does movement in the opposite direction (see also Krumhansl, 1979, 1990).

Effects of pitch register and pitch direction were observed not only for untrained listeners, but also for trained listeners. It has also been found that estimates of interval size by trained listeners are influenced by timbre (Russo \& Thompson, 2005). Insofar as trained listeners can classify and label melodic intervals, these findings suggest a distinction between the distance-quality of pitch intervals and the analytic labels associated with those intervals.

\section{CONCLUSION}

Models of melodic perception often draw distinctions about whether an interval is small or large or whether a sequence of intervals proceeds from small to large or from large to small (Deliège, 1987; Jones, 1987; Larson \& McAdams, 2004; Lerdahl, 1989; Lerdahl \& Jackendoff, 1983; Margulis, 2005; Narmour, 1990). The relation between the physical and the perceived sizes of an interval is dependent on its pitch register and pitch direction, as well as on the experience of the listener. Thus, intervals that are disparate in physical size can, in some contexts, be similar in perceived interval size. As is documented 
in the Appendix, for example, an ascending interval of seven semitones presented in the low pitch register was perceived by untrained listeners as roughly equivalent in size to a descending interval of three semitones (regardless of pitch register). Given that seven semitones generally is understood to be a large interval and three semitones a small interval, these scales have important implications for predictions about the effects of interval size. In particular, they may be used in place of the conventional (logarithmic) scale, increasing the specificity of predictions about such issues as the perception of accent or grouping structure, melodic expectancies, and a range of other auditory phenomena, both musical and nonmusical (e.g., prosody and auditory display).

\section{REFERENCES}

Attneave, F., \& Olson, R. K. (1971). Pitch as a medium: A new approach to psychophysical scaling. American Journal of Psychology, 84, 147-166.

Bartlett, J. C. (1993). Tonal structure of melodies. In J. T. Tighe \& W. J. Dowling (Eds.), Psychology and music: The understanding of melody and rhythm (pp. 39-61). Hillsdale, NJ: Erlbaum.

BECK, J., \& SHAW, W. A. (1961). The scaling of pitch by the method of magnitude estimation. American Journal of Psychology, 74, 242-251.

BharUCha, J. J., \& PrYOR, J. H. (1986). Disrupting the isochrony underlying rhythm: An asymmetry in discrimination. Perception \& Psychophysics, 40, 137-141.

Boersma, P., \& Weenink, D. (2004). Praat: Doing phonetics by computer. Available at http://www.praat.org/.

Boltz, M., \& Jones, M. R. (1986). Does rule recursion make melodies easier to reproduce? If not, what does? Cognitive Psychology, 18, 389-431.

Bregman, A. S. (1990). Auditory scene analysis: The perceptual organization of sound. Cambridge, MA: MIT Press.

Burns, E. M. (1999). Intervals, scales, and tuning. In D. Deutsch (Ed.), The psychology of music (2nd ed., pp. 215-264). New York: Academic Press.

Burns, E. M., \& Campbell, S. L. (1994). Frequency and frequencyratio resolution by possessors of absolute and relative pitch: Examples of categorical perception? Journal of the Acoustical Society of America, 96, 2704-2719.

Burns, E. M., \& WARD, W. D. (1978). Categorical perceptionphenomenon or epiphenomenon: Evidence from experiments in the perception of melodic musical intervals. Journal of the Acoustical Society of America, 63, 456-468.

DelièGe, I. (1987). Grouping conditions in listening to music: An approach to Lerdahl and Jackendoff's grouping preference rules. Music Perception, 4, 325-359.

Deutsch, D. (1969). Music recognition. Psychological Review, 76, 300307.

Deutsch, D. (1972). Octave generalization and tune recognition. Perception \& Psychophysics, 11, 411-412.

Deutsch, D. (1978a). Delayed pitch comparisons and the principle of proximity. Perception \& Psychophysics, 23, 227-230.

Deutsch, D. (1978b). Pitch memory: An advantage for the left-handed. Science, 199, 559-560.

Deutsch, D., \& Boulanger, R. C. (1984). Octave equivalence and the immediate recall of pitch sequences. Music Perception, 2, 40-51.

Drake, C., Dowling, W. J., \& Palmer, C. (1991). Accent structures in the reproduction of simple tunes by children and adult pianists. Music Perception, 8, 315-334.

Greenwood, D. D. (1997). The Mel Scale's disqualifying bias and a consistency of pitch-difference equisections in 1956 with equal cochlear distances and equal frequency ratios. Hearing Research, 103, 199-224.

Helson, H. (1964). Adaptation-level theory: An experimental and systematic approach to behavior. New York: Harper \& Row.
Huron, D. (2001). Tone and voice: A derivation of the rules of voiceleading from perceptual principles. Music Perception, 19, 1-64.

Jones, M. R. (1987). Dynamic pattern structure in music: Recent theory and research. Perception \& Psychophysics, 41, 621-634.

Killam, R. N., Lorton, P. V., JR., \& Schubert, E. D. (1975). Interval recognition: Identification of harmonic and melodic intervals. Journal of Music Theory, 19, 212-234.

KRUMHANSL, C. L. (1979). The psychological representation of musical pitch in a tonal context. Cognitive Psychology, 11, 346-374.

Krumhansl, C. L. (1990). Cognitive foundations of musical pitch. London: Oxford University Press.

Krumhansl, C. L. (2000). Rhythm and pitch in music cognition. Psychological Bulletin, 126, 159-179.

LARSON, S., \& McAdAMs, S. (2004). Musical forces and melodic expectations: Comparing computer models and experimental results. Music Perception, 21, 457-498.

Lerdahl, F. (1989). Atonal prolongational structure. Contemporary Music Review, 4, 65-87.

LERDAHL, F., \& JACKENDOFF, R. (1983). A generative theory of tonal music. Cambridge, MA: MIT Press.

LEWIS, D. (1942). Pitch scales. Journal of the Acoustical Society of America, 14, 127.

MaKeIG, S. (1982). Affective versus analytic perception of musical intervals. In M. Clynes (Ed.), Music, mind, and brain: The neuropsychology of music (pp. 227-250). New York: Plenum.

Margulis, E. H. (2005). A model of melodic expectation. Music Perception, 22, 663-714.

Marley, A. A. J., \& CooK, V. T. (1984). A fixed rehearsal capacity interpretation of limits on absolute identification performance. British Journal of Mathematical \& Statistical Psychology, 37, 136-151.

Meyer, L. B. (1973). Explaining music: Essays and explorations. Berkeley: University of California Press.

NARMOUR, E. (1990). The analysis and cognition of basic melodic structures: The implication-realization model. Chicago: University of Chicago Press.

Plomp, R., WagenaAr, W., \& Mimpen, A. (1973). Musical interval recognition with simultaneous tones. Acustica, 29, 101-109.

Rasch, R., \& Plomp, R. (1999). The perception of musical tones. In D. Deutsch (Ed.), The psychology of music (2nd ed., pp. 89-112). New York: Academic Press.

Rosch, E. (1975). Cognitive reference points. Cognitive Psychology, 7, 532-547.

Russo, F. A. (2002). The influence of bottom-up expectancy on melodic processing difficulty. Unpublished doctoral dissertation, Queen's University, Kingston, ON.

Russo, F. A., \& Thompson, W. F. (2005). An interval size illusion: The influence of timbre on the perceived size of melodic intervals. Perception \& Psychophysics, 67, 559-568.

SchellenberG, E. G. (2001). Asymmetries in the discrimination of musical intervals: Going out-of-tune is more noticeable than going in-tune. Music Perception, 19, 223-248.

Schneider, B., Parker, S., \& Upenieks, E. G. (1982). The perceptual basis of judgments of pitch differences and pitch ratios. Canadian Journal of Psychology, 36, 4-23.

Shepard, R. (1999). Pitch perception and measurement. In P. R. Cook (Ed.), Music, cognition, and computerized sound: An introduction to psychoacoustics (pp. 149-165). Cambridge, MA: MIT Press.

Siegel, J. A., \& Siegel, W. (1977a). Absolute identification of notes and intervals by musicians. Perception \& Psychophysics, 21, 143-152.

Siegel, J. A., \& Siegel, W. (1977b). Categorical perception of tonal intervals: Musicians can't tell sharp from flat. Perception \& Psychophysics, 21, 399-407.

SiEgEL, R. J. (1964). A replication of the mel scale of pitch. American Journal of Psychology, 78, 615-620.

Stevens, S. S., \& Volkmann, J. (1940). The relation of pitch to frequency: A revised scale. American Journal of Psychology, 53, 329353.

Stevens, S. S., Volkmann, J., \& Newman, E. B. (1937). A scale for the measurement of the psychological magnitude pitch. Journal of the Acoustical Society of America, 8, 185-190.

StumpF, C. (1883). Tonpsychologie (Vol. 1). Leipzig: Hirzel. 
Titchener, E. B. (1905). Experimental psychology (Vol. 2, Pt. 2). New York: Macmillan.

Tversky, A., \& Gati, I. (1978). Studies of similarity. In E. Rosch \& B. Lloyd (Eds.), Cognition and categorization (pp. 79-98). Hillsdale, NJ: Erlbaum.

VON HIPPEL, P., \& HURON, D. (2000). Why do skips precede reversals? The effect of tessitura on melodic structure. Music Perception, 18, $59-85$.

Vos, P. G., \& Troost, J. M. (1989). Ascending and descending melodic intervals: Statistical findings and their perceptual relevance. Music Perception, 6, 383-396.

\section{NOTES}

1. We performed a secondary analysis of slopes to assess whether compatibility of intervals with the chromatic scale influenced estimates. Half of the intervals tested are compatible with a chromatic scale (100 cents, 200 cents, 300 cents, etc.), and half are not (50 cents, 150 cents, 250 cents, etc.). Slopes were determined (as described above) for chromatic and nonchromatic intervals up to an octave and larger than an octave. These slopes were subjected to a mixed-design ANOVA with training as the between-subjects variable and with chromatic scale (compatible or incompatible) and interval set (small or large) as the within-subjects variables. The mean slope for chromatic intervals was nearly identical to the mean slope for nonchromatic intervals $[M=2.8$ and 2.84, respectively; $F(1,26)<1]$. Moreover, none of the interactions involving chromatic scale was significant.

2. Although its well established that long-term pitch distributional information is internalized by listeners and that it has strong influence over perceptual judgments, it is also possible that pitch distributional information yielded by the experimental design (i.e., central pitches occurred more frequently) may have led to short-term expectancies that favored movement toward the center of the established pitch range. Denial of these short-term expectancies may have contributed to larger estimates of interval size. However, the influence of short-term expectancies should have increased only as a participant progressed through the four blocks of the experiment and became more familiar with its pitch distributional information. In a reanalysis of interval size estimates made in the initial block of trials, we found that means for ascending and descending intervals in the high and low pitch registers were nearly identical to the corresponding overall means (all differences were within $5 \%)$. This finding is consistent with the suggestion that the observed interaction between pitch direction and pitch register was due primarily to long-term expectancies for movement toward the mean pitch. 


\section{APPENDIX}

Scales of Interval Size for Different Levels of Pitch Register and Pitch Direction at Different Levels of Musical Experience

\begin{tabular}{|c|c|c|c|c|c|c|c|c|}
\hline \multirow[b]{3}{*}{ Semitones } & \multicolumn{4}{|c|}{ Trained } & \multicolumn{4}{|c|}{ Untrained } \\
\hline & \multicolumn{2}{|c|}{ Ascending } & \multicolumn{2}{|c|}{ Descending } & \multicolumn{2}{|c|}{ Ascending } & \multicolumn{2}{|c|}{ Descending } \\
\hline & High & Low & High & Low & High & Low & High & Low \\
\hline 0.5 & 2.24 & 7.61 & 5.60 & 4.12 & 10.11 & 10.85 & 16.81 & 15.19 \\
\hline 1.0 & 5.53 & 9.47 & 8.08 & 7.04 & 12.61 & 11.95 & 18.09 & 17.08 \\
\hline 1.5 & 8.75 & 11.30 & 10.50 & 9.91 & 15.07 & 13.04 & 19.38 & 18.94 \\
\hline 2.0 & 11.90 & 13.09 & 12.88 & 12.73 & 17.49 & 14.13 & 20.66 & 20.77 \\
\hline 2.5 & 14.99 & 14.84 & 15.20 & 15.48 & 19.87 & 15.22 & 21.94 & 22.57 \\
\hline 3.0 & 18.01 & 16.56 & 17.49 & 18.18 & 22.21 & 16.30 & 23.23 & 24.35 \\
\hline 3.5 & 20.96 & 18.25 & 19.72 & 20.82 & 24.52 & 17.37 & 24.51 & 26.09 \\
\hline 4.0 & 23.85 & 19.89 & 21.90 & 23.41 & 26.78 & 18.44 & 25.79 & 27.81 \\
\hline 4.5 & 26.67 & 21.50 & 24.04 & 25.93 & 29.00 & 19.50 & 27.08 & 29.50 \\
\hline 5.0 & 29.42 & 23.08 & 26.13 & 28.41 & 31.18 & 20.56 & 28.36 & 31.16 \\
\hline 5.5 & 32.11 & 24.62 & 28.17 & 30.82 & 33.32 & 21.61 & 29.65 & 32.79 \\
\hline 6.0 & 34.73 & 26.12 & 30.17 & 33.18 & 35.42 & 22.66 & 30.93 & 34.40 \\
\hline 6.5 & 37.29 & 27.59 & 32.11 & 35.48 & 37.48 & 23.70 & 32.21 & 35.97 \\
\hline 7.0 & 39.77 & 29.02 & 34.01 & 37.72 & 39.50 & 24.73 & 33.50 & 37.52 \\
\hline 7.5 & 42.20 & 30.41 & 35.86 & 39.90 & 41.49 & 25.76 & 34.78 & 39.04 \\
\hline 8.0 & 44.55 & 31.77 & 37.67 & 42.03 & 43.43 & 26.79 & 36.07 & 40.53 \\
\hline 8.5 & 46.84 & 33.09 & 39.42 & 44.10 & 45.33 & 27.81 & 37.35 & 41.99 \\
\hline 9.0 & 49.07 & 34.38 & 41.13 & 46.12 & 47.19 & 28.82 & 38.63 & 43.42 \\
\hline 9.5 & 51.22 & 35.63 & & & 49.01 & 29.83 & & 44.83 \\
\hline 10.0 & 53.31 & 36.85 & 44.41 & 49.98 & 50.80 & 30.83 & 41.20 & 46.20 \\
\hline 10.5 & 55.34 & 38.02 & & 51.82 & 52.54 & 31.83 & 42.48 & 47.55 \\
\hline 11.0 & 57.29 & 39.17 & 47.49 & 53.61 & 54.24 & 32.82 & 43.77 & 48.87 \\
\hline 11.5 & 59.18 & 40.27 & 48.96 & 55.34 & 55.90 & 33.81 & 45.05 & 50.16 \\
\hline 12.0 & 61.01 & 41.35 & 50.38 & 57.01 & 57.53 & 34.79 & 46.33 & 51.42 \\
\hline 12.5 & 62.77 & 42.38 & 51.76 & 58.63 & 59.11 & 35.77 & 47.62 & 52.66 \\
\hline 13.0 & 64.46 & 43.38 & 53.08 & 60.19 & 60.65 & 36.74 & 48.90 & 53.86 \\
\hline 13.5 & 66.09 & 44.34 & 54.36 & 61.69 & 62.15 & 37.71 & 50.19 & 55.04 \\
\hline 14.0 & 67.64 & 45.27 & 55.59 & 63.13 & 63.62 & 38.67 & 51.47 & 56.18 \\
\hline 14.5 & 69.14 & 46.16 & 56.78 & 64.52 & 65.04 & 39.62 & 52.75 & 57.30 \\
\hline 15.0 & 70.56 & 47.01 & 57.91 & 65.85 & 66.42 & 40.57 & 54.04 & 58.39 \\
\hline 15.5 & 71.92 & 47.83 & 59.00 & 67.12 & 67.77 & 41.51 & 55.32 & 59.46 \\
\hline 16.0 & 73.22 & 48.62 & 60.04 & 68.34 & 69.07 & 42.45 & 56.60 & 60.49 \\
\hline 16.5 & 74.44 & 49.36 & 61.03 & 69.50 & 70.33 & 43.39 & 57.89 & 61.50 \\
\hline 17.0 & 75.60 & 50.07 & 61.98 & 70.60 & 71.56 & 44.31 & 59.17 & 62.47 \\
\hline 17.5 & 76.70 & 50.75 & 62.88 & 71.65 & 72.74 & 45.24 & 60.45 & 63.42 \\
\hline 18.0 & 77.73 & 51.39 & 63.73 & 72.64 & 73.89 & 46.15 & 61.74 & 64.34 \\
\hline 18.5 & 78.69 & 51.99 & 64.53 & 73.57 & 74.99 & 47.06 & 63.02 & 65.23 \\
\hline 19.0 & 79.58 & 52.56 & 65.28 & 74.44 & 76.05 & 47.97 & 64.30 & 66.10 \\
\hline 19.5 & 80.41 & 53.09 & 65.99 & 75.26 & 77.08 & 48.87 & 65.59 & 66.93 \\
\hline 20.0 & 81.17 & 53.58 & 66.65 & 76.02 & 78.06 & 49.77 & 66.87 & 67.74 \\
\hline 20.5 & 81.87 & 54.04 & 67.26 & 76.73 & 79.01 & 50.66 & 68.16 & 68.51 \\
\hline 21.0 & 82.50 & 54.46 & 67.82 & 77.37 & 79.91 & 51.54 & 69.44 & 69.26 \\
\hline 21.5 & 83.06 & 54.85 & 68.34 & 77.96 & 80.77 & 52.42 & 70.72 & 69.98 \\
\hline 22.0 & 83.56 & 55.20 & 68.81 & 78.50 & 81.60 & 53.29 & 72.01 & 70.67 \\
\hline 22.5 & 83.99 & 55.52 & 69.23 & 78.97 & 82.38 & 54.16 & 73.29 & 71.34 \\
\hline 23.0 & 84.35 & 55.80 & 69.60 & 79.39 & 83.13 & 55.03 & 74.57 & 71.97 \\
\hline 23.5 & 84.65 & 56.04 & 69.92 & 79.75 & 83.83 & 55.88 & 75.86 & 72.58 \\
\hline 24.0 & 84.88 & 56.25 & 70.20 & 80.06 & 84.50 & 56.73 & 77.14 & 73.16 \\
\hline
\end{tabular}

(Manuscript received May 20, 2004;

revision accepted for publication March 2, 2005.) 